Preprint typeset in JHEP style - HYPER VERSION

SHEP 01-28

\title{
Exact renormalization group equation in presence of rescaling anomaly II - The local potential approximation
}

\author{
S. Arnone ${ }^{a}$, D. Francia ${ }^{b}$ and K. Yoshida ${ }^{b}$ \\ ${ }^{a}$ Department of Physics and Astronomy, University of Southampton \\ Highfield, Southampton SO17 1BJ, U.K. \\ ${ }^{b}$ Dipartimento di Fisica, Università degli Studi di Roma "La Sapienza" \\ P.le Aldo Moro, 2 - 00185 Roma, Italia and I.N.F.N., Sezione di Roma I \\ E-mails: sa@hep.phys.soton.ac.uk, dario.francia@roma1.infn.it, \\ kensuke.yoshida@roma1.infn.it
}

\begin{abstract}
Exact renormalization group techniques are applied to mass deformed $\mathcal{N}=4$ supersymmetric Yang-Mills theory, viewed as a regularised $\mathcal{N}=2$ model. The solution of the flow equation, in the local potential approximation, reproduces the one-loop (perturbatively exact) expression for the effective action of $\mathcal{N}=2$ supersymmetric Yang-Mills theory, when the regularising mass, $M$, reaches the value of the dynamical cutoff $\Lambda$. One speculates about the way in which further non-perturbative contributions (instanton effects) may be accounted for.
\end{abstract}




\section{Contents}

1. Introduction 1

2. Exact renormalization group equation for SUSY gauge theories 2

3. The local potential approximation to Polchinski's equation 5

3.1 Generalisation to $S U(N)$

4. Conclusion and comments 11

\section{Introduction}

In a previous paper [1], we have studied the proposals by Arkani-Hamed and Murayama [2, 3] concerning the renormalization group $(\mathrm{RG})$ invariance of exact results in supersymmetric (SUSY) gauge field theories. Our main tool was the method of the exact renormalization group (ERG) equation [1], inspired by the decimation method by Wilson [4] and adapted by Polchinski and Gallavotti [5] to the continuum field theory case.

The main results in [1] are:

- the dominant part of the low energy effective (a.k.a. "Wilsonian") action which is responsible for the Novikov-Shifman-Vainshtein-Zakharov (NSVZ) exact expression for the beta function [6] is the anomalous term due to the rescaling (a.k.a. Konishi) anomaly [7];

- after subtracting such an anomalous term, the remainder of the effective action satisfies the ERG equation, or Polchinski's equation, with respect to the variation of the regularising mass, $M$.

After these results, as a further test of our method, we would like to see whether it can be used to get further useful information about the low energy interactions of SUSY gauge field theories, beyond the NSVZ formula.

In particular, it should be possible, at least in principle, to compute the low energy effective action for the $\mathcal{N}=2$ SUSY Yang-Mills theory (SYM), as regularised as the ultraviolet (UV) finite mass deformed $\mathcal{N}=4 \mathrm{SYM}[8]$.

In a subsequent note 9], however, we adopted the idea that the detailed structure of low energy effective action could be obtained by generalising the "anomaly analysis" in [1, 5] rather than seeking the solution of the ERG equation we derived in [1]. As a matter of fact, in [9] it has been tacitly assumed that the "non-anomalous" part of the effective action becomes irrelevant once the generalised Konishi anomaly has been introduced.

The expression of the low energy effective action (the vector field kinematical term) of $\mathcal{N}=2 \mathrm{SYM}$ given in [9], although similar in functional form to the standard perturbately exact one-loop result, has the wrong dependence on the relevant mass parameters $(M$ and 
$M_{0}$, the latter being the starting point of the flow), so that one cannot reproduce the correct exclusive dependence on the dynamical cutoff $\Lambda$.

In the present note, we would like to pursue the original idea in [1] and look back at the ERG equation for the non-anomalous part of the effective action. We will try and solve that equation in the simplest standard approximation, i.e. the local potential approximation (LPA).

It might be expected that, within such approximation, one would essentially end up with the same result as in [9]. Instead, the effective action we computed shows similar functional dependence on the chiral superfield $\varphi$ but different dependence on the mass parameters. This change brings the new result into complete agreement with the standard perturbately exact result for $\mathcal{N}=2 \mathrm{SYM}$ in weak coupling regime.

In [1], Polchinski's original derivation of the ERG equation has been closely followed. With this method one sees the appearance of a singular functional derivative term which is then identified with the contribution of the rescaling anomaly. Even though this passage can be made more convincing by using some pre-regularisation, it would be nice to avoid any reference to such a singular term. This can be achieved by applying the method proposed by $\mathrm{C}$. Becchi [10]. In the standard RG approach, this method consists in damping high modes by a suitable field redefinition, $\phi_{p} \rightarrow K_{\Lambda} \phi_{p}$, in the interaction part of the action only. Thus, up to a vacuum energy term, it is equivalent to modifying the free propagator, $D(p) \rightarrow D(p) K_{\Lambda}^{-1} \cdot K_{\Lambda} \equiv K\left(\frac{p^{2}}{\Lambda^{2}}\right)$ is a smooth ultraviolet cutoff profile, rapidly vanishing at infinity.

The paper is organised as follows: in sec. 2 we introduce the mass deformed $\mathcal{N}=4 \mathrm{SYM}$ theory and re-derive the ERG equation in presence of the rescaling anomaly by generalising Becchi's transformation. Sec. 3 is devoted to the study of the flow equation in the LPA: we start off with the $S U(2)$ case and, then, generalise the results to $S U(N)$. In sec. 4 we summarise and draw our conclusions.

\section{Exact renormalization group equation for SUSY gauge theories}

As in [1], we consider the mass deformed version of $\mathcal{N}=4 S U(N)$ SYM in four space-time dimensions, corresponding to the so-called $\mathcal{N}=1^{*}$ or $\mathcal{N}=2^{*}$ models.

The classical action is given by $S^{*}=S_{\mathcal{N}=4}+$ mass terms and reads (written in terms of $\mathcal{N}=1$ superfields and in the "holomorphic" representation)

$$
\begin{aligned}
& S^{*}\left(V, \varphi_{i}, \bar{\varphi}_{i} ; g_{0}\right)=\frac{1}{16} \int d^{4} x d^{2} \theta \frac{1}{g_{0}^{2}} W_{\alpha}^{a} W^{a \alpha}+\int d x d^{4} \theta \Re e\left(\frac{2}{g_{0}^{2}}\right) N \sum_{i=1}^{3} \bar{\varphi}_{i} e^{V} \varphi_{i}+ \\
& +\int d^{4} x d^{2} \theta \Re e\left(\frac{\sqrt{2}}{g_{0}^{2}}\right) i N f_{a b c} \varphi_{a}^{i} \varphi_{b}^{j} \varphi_{c}^{k} \frac{\epsilon^{i j k}}{3 !}+\frac{1}{2} \int d^{4} x d^{2} \theta \sum_{i=1}^{\mu} M_{i}^{0} \varphi_{i}^{2}+h . c .
\end{aligned}
$$

all the relevant fields transforming as the adjoint representation of the gauge group.

In order to break supersymmetry down to $\mathcal{N}=2$ or $\mathcal{N}=1$, one gives mass to some of the chiral superfields in the $\mathcal{N}=4$ supermultiplet. These masses are supposed be large compared to the range of momenta one is interested in. In what follows we will consider the 
case in which two chiral superfields are given the same mass, i.e. $\mu=2$ and $M_{1}^{0}=M_{2}^{0}=M_{0}$, as it preserves $\mathcal{N}=2$ SUSY.

This model, just as the original $\mathcal{N}=4$ SYM theory without chiral mass terms, is believed to be finite. It has been shown that, at the perturbative level, all the UV divergences cancel out [8].

The generating functional takes the form

$$
\begin{aligned}
& Z_{M_{0}}=\int \mathcal{D}[V] \mathcal{D}[\varphi] \mathcal{D}[\bar{\varphi}] \prod_{i=1}^{2} \mathcal{D}\left[\varphi_{i}\right] \mathcal{D}\left[\bar{\varphi}_{i}\right] \exp i\left[S_{\mathcal{N}=4}\left(V, \varphi, \bar{\varphi}, \varphi_{i}, \bar{\varphi}_{i} ; g_{0}\right)+\right. \\
& \left.+\frac{M_{0}}{2} \int \sum_{i=1}^{2} \varphi_{i}^{2}+\text { h.c. }+\int \sum_{i=1}^{2} J_{i} \varphi_{i}+\text { h.c. }+\int J_{\varphi} \varphi+\int J_{\bar{\varphi}} \bar{\varphi}+\int J_{V} V\right]
\end{aligned}
$$

where $\varphi$ is the massless chiral superfield before denoted by $\varphi_{3}$ and the integration over the chiral or full superspace has not been written explicitly. ${ }^{1}$ The field set $(V, \varphi, \bar{\varphi})$ is often referred to as the $\mathcal{N}=2$ vector multiplet.

The ERG method consists in varying the regularising mass $M_{0}$ to $M \leq M_{0}$ and compensating for such change by replacing the bare action by the Wilsonian effective action $S_{M}$, i.e.

$$
\begin{aligned}
& Z_{M_{0}}=Z_{M} \equiv \int \mathcal{D}[V] \mathcal{D}[\varphi] \mathcal{D}[\bar{\varphi}] \prod_{i=1}^{2} \mathcal{D}\left[\varphi_{i}\right] \mathcal{D}\left[\bar{\varphi}_{i}\right] \exp i\left[S_{M}\left(V, \varphi, \bar{\varphi}, \varphi_{i}, \bar{\varphi}_{i}\right)+\right. \\
& \left.+\frac{M}{2} \int \sum_{i=1}^{2} \varphi_{i}^{2}+\int \sum_{i=1}^{2} f(M) J_{i} \varphi_{i}+\frac{1}{2} \int \sum_{i=1}^{2} g(M) J_{i}^{2}+\int J_{\varphi} \varphi+h . c .+\int J_{V} V\right] .
\end{aligned}
$$

Demanding the generating functional be invariant under the continuous change in the parameter $M$ one gets the equation obeyed by the effective action $S_{M}$.

Following the method in [10], we implement Becchi's transformation by rescaling auxiliary chiral superfields,

$$
\varphi_{i}=\sqrt{\frac{M_{0}}{M}} \varphi_{i}^{\prime}, \quad \quad \bar{\varphi}_{i}=\sqrt{\frac{M_{0}}{M}} \bar{\varphi}_{i}^{\prime}, \quad i=1,2 .
$$

In this way, the dependence upon $M$ is transferred from the mass term to the effective action itself. Moreover, the functional measure for the relevant fields is known to acquire the non-trivial Jacobian determinant []]

$$
\prod_{i=1}^{\mu} \mathcal{D}\left[\varphi_{i}\right] \mathcal{D}\left[\bar{\varphi}_{i}\right]=\prod_{i=1}^{\mu} \mathcal{D}\left[\varphi_{i}^{\prime}\right] \mathcal{D}\left[\bar{\varphi}_{i}^{\prime}\right] \exp \left[-\frac{i}{2} \int d^{4} x d^{2} \theta \frac{\mu N}{64 \pi^{2}} \log \left(\frac{M}{M_{0}}\right) W_{\alpha}^{a} W^{a \alpha}+\text { h.c. }\right]
$$

where $\mu=2$ in the present case and the factor $-\frac{1}{2}$ is due to $\log \sqrt{\frac{M_{0}}{M}}=-\frac{1}{2} \log \left(\frac{M}{M_{0}}\right)$.

\footnotetext{
${ }^{1}$ In order to simplify notation, we will dispense with the integration measure in what follows.
} 
Thus $Z_{M}$ can be rewritten as

$$
\begin{aligned}
& Z_{M}=\int \mathcal{D}[V] \mathcal{D}[\varphi] \mathcal{D}[\bar{\varphi}] \prod_{i=1}^{2} \mathcal{D}\left[\varphi_{i}^{\prime}\right] \mathcal{D}\left[\bar{\varphi}_{i}^{\prime}\right] \exp i\left[S_{M}\left(V, \varphi, \bar{\varphi}, \sqrt{\frac{M_{0}}{M}} \varphi_{i}^{\prime}, \sqrt{\frac{M_{0}}{M}} \bar{\varphi}_{i}^{\prime}\right)+\right. \\
& -\frac{N}{64 \pi^{2}} \int \log \left(\frac{M}{M_{0}}\right) W_{\alpha}^{a} W^{a \alpha}+\text { h.c. }+\frac{M_{0}}{2} \int \sum_{i=1}^{2} \varphi_{i}^{\prime 2}+\int \sum_{i=1}^{2} f(M) \sqrt{\frac{M_{0}}{M}} J_{i} \varphi_{i}^{\prime}+ \\
& \left.+\frac{1}{2} \int \sum_{i=1}^{2} g(M) J_{i}^{2}+\text { h.c. }+\int J_{\varphi} \varphi+\int J_{\bar{\varphi}} \bar{\varphi}+\int J_{V} V\right] .
\end{aligned}
$$

As pointed out before, the equation for $S_{M}$ follows from the invariance of the generating functional, $Z_{M_{0}}=Z_{M}$, or equivalently $M \partial_{M} Z_{M}=0$.

$$
\begin{aligned}
& M \partial_{M} Z_{M}=i \int \mathcal{D}[V] \mathcal{D}[\varphi] \mathcal{D}[\bar{\varphi}] \prod_{i=1}^{2} \mathcal{D}\left[\varphi_{i}^{\prime}\right] \mathcal{D}\left[\bar{\varphi}_{i}^{\prime}\right]\left[-\frac{N}{64 \pi^{2}} \int W_{\alpha}^{a} W^{a \alpha}+\text { h.c. }+\right. \\
& +\left.M \partial_{M} S_{M}\right|_{\varphi_{i}, \bar{\varphi}_{i}}+M \partial_{M} \sqrt{\frac{M_{0}}{M}} \int \varphi_{i}^{\prime} \frac{\delta S_{M}}{\delta \varphi_{i}}+M \partial_{M} \sqrt{\frac{M_{0}}{M}} \int \bar{\varphi}_{i}^{\prime} \frac{\delta S_{M}}{\delta \bar{\varphi}_{i}}+ \\
& \left.+M \partial_{M} \int f(M) \sqrt{\frac{M_{0}}{M}} J_{i} \varphi_{i}^{\prime}+\frac{1}{2} M \partial_{M} \int g(M) J_{i}^{2}+\text { h.c. }\right] \exp i S_{t o t},
\end{aligned}
$$

where $S_{\text {tot }}$ stands for the argument of the exponential in eq. (2.4) and the sum upon $i$ has been left out.

Noting that the equation of motion $\varphi_{i}^{\prime}=\frac{1}{M_{0}}\left(\frac{\delta S_{t o t}}{\delta \varphi_{i}^{\prime}}-\frac{\delta S_{M}}{\delta \varphi_{i}^{\prime}}-f(M) \sqrt{\frac{M_{0}}{M}} J_{i}\right)$ is valid within the functional integral, the r.h.s. of eq. (2.5) can be recast as

$$
\begin{aligned}
& i \int \mathcal{D}[V] \mathcal{D}[\varphi] \mathcal{D}[\bar{\varphi}] \prod_{i=1}^{2} \mathcal{D}\left[\varphi_{i}^{\prime}\right] \mathcal{D}\left[\bar{\varphi}_{i}^{\prime}\right]\left[-\frac{N}{64 \pi^{2}} \int W_{\alpha}^{a} W^{a \alpha}+\text { h.c. }+\left.M \partial_{M} S_{M}\right|_{\varphi_{i}, \bar{\varphi}_{i}}+\right. \\
& -\frac{1}{2 M} \int \frac{\delta S_{M}}{\delta \varphi_{i}}\left(\frac{\delta S_{t o t}}{\delta \varphi_{i}^{\prime}}-\frac{\delta S_{M}}{\delta \varphi_{i}^{\prime}}-f(M) \sqrt{\frac{M_{0}}{M}} J_{i}\right)+M \partial_{M} \int f(M) \sqrt{\frac{M_{0}}{M}} J_{i} \varphi_{i}^{\prime}+ \\
& \left.+\frac{1}{2} M \partial_{M} \int g(M) J_{i}^{2}\right] \exp i S_{t o t} .
\end{aligned}
$$

Integrating by parts, so that $\frac{\delta S_{M}}{\delta \varphi_{i a}} \frac{\delta S_{t o t}}{\delta \varphi_{i a}}$ becomes $i \frac{\delta^{2} S_{M}}{\delta \varphi_{i a} \delta \varphi_{i a}}$, and making again use of the above equation of motion, eq. (2.6) becomes

$$
\begin{aligned}
& i \int \mathcal{D}[V] \mathcal{D}[\varphi] \mathcal{D}[\bar{\varphi}] \prod_{i=1}^{2} \mathcal{D}\left[\varphi_{i}^{\prime}\right] \mathcal{D}\left[\bar{\varphi}_{i}^{\prime}\right]\left[\left.M \partial_{M} S_{M}\right|_{\varphi_{i}, \bar{\varphi}_{i}}-\frac{1}{2 M} \int\left(i \frac{\delta^{2} S_{M}}{\delta \varphi_{i} \delta \varphi_{i}}-\frac{\delta S_{M}}{\delta \varphi_{i}}\right)+\right. \\
& -\frac{1}{2 M} \int\left(\sqrt{M_{0} M} \varphi_{i}^{\prime}+f(M) J_{i}\right) f(M) J_{i}+M \partial_{M} \int f(M) \sqrt{\frac{M_{0}}{M}} J_{i} \varphi_{i}^{\prime}+ \\
& \left.+\frac{1}{2} M \partial_{M} \int g(M) J_{i}^{2}-\frac{N}{64 \pi^{2}} \int W_{\alpha}^{a} W^{a \alpha}+\text { h.c. }\right] \exp i S_{t o t} .
\end{aligned}
$$

The equations for $f(M), g(M)$ simply follow from the inspection of eq. (2.7),

$$
M \partial_{M} f(M)=f(M), \quad M \partial_{M} g(M)=\frac{1}{M} f^{2}(M) .
$$


The solutions to the above equations with the proper boundary conditions $-f\left(M_{0}\right)=1$ and $g\left(M_{0}\right)=0$ - are $f(M)=\frac{M}{M_{0}}$ and $g(M)=\frac{M}{M_{0}^{2}}-\frac{1}{M_{0}}$.

Hence the generating functional is indeed invariant under the RG transformation if the effective action satisfies the anomalous Polchinski's equation

$$
M \partial_{M} S_{M}=\frac{1}{2 M} \int\left[i \frac{\delta^{2} S_{M}}{\delta \varphi_{i} \delta \varphi_{i}}-\frac{\delta S_{M}}{\delta \varphi_{i}} \frac{\delta S_{M}}{\delta \varphi_{i}}\right]+\text { h.c. }+\frac{N}{64 \pi^{2}} \int W_{\alpha}^{a} W^{a \alpha}+\text { h.c. }
$$

As in [1], the above equation can be also recast as

$$
M \partial_{M} \tilde{S}_{M}=\frac{1}{2 M} \int\left[i \frac{\delta^{2} \tilde{S}_{M}}{\delta \varphi_{i} \delta \varphi_{i}}-\frac{\delta \tilde{S}_{M}}{\delta \varphi_{i}} \frac{\delta \tilde{S}_{M}}{\delta \varphi_{i}}\right]+\text { h.c. }
$$

where $\tilde{S}_{M}=S_{M}-\frac{N}{64 \pi^{2}} \int \log \left(\frac{M}{M_{0}}\right) W_{\alpha}^{a} W^{a \alpha}+$ h.c., as the contribution of the anomaly, $\tilde{S}_{M}-S_{M}$, does not depend upon the auxiliary massive fields.

Eq. (2.8) is the main result in [1]. As stated in the introduction, in the present derivation singular terms such as $\sum_{i} \frac{\delta \varphi_{i}}{\delta \varphi_{i}}$ do not appear at all. ${ }^{2}$

\section{The local potential approximation to Polchinski's equation}

As outlined in the introduction, we would like to find the solution of eq. (2.8) within some approximation scheme, different from the standard perturbative loop expansion. For the relevant initial condition at $M=M_{0}$, we will try and determine $\tilde{S}_{M}$ at the lowest order approximation in the derivative expansion, i.e. the local potential approximation.

In order to carry out the LPA consistently, one usually first Legendre transforms the Wilsonian action $S_{\Lambda}$ ( $\Lambda$ being the cutoff, in the present case the regularising mass parameter) to the one-particle-irreducible action $\Gamma_{\Lambda \Lambda_{0}}$ [11], with $\Lambda$ now acting as an infrared cutoff, and then applies the Coleman-Weinberg expansion

$$
\Gamma_{\Lambda \Lambda_{0}}(\phi)=\int d^{4} x V_{\Lambda \Lambda_{0}}(\phi)+\int d^{4} x Z_{\Lambda \Lambda_{0}}(\phi)\left(\partial_{\mu} \phi\right)^{2}+\cdots,
$$

with the ellipsis standing for higher derivative terms. Substituting $\Gamma_{\Lambda \Lambda_{0}}(\phi)$ back into the flow equation, the various coefficients, $V_{\Lambda \Lambda_{0}}(\phi), Z_{\Lambda \Lambda_{0}}(\phi), \ldots$, can be consistently determined [11].

In the present note, since we are interested in the lowest order local potential term in a range of momenta much lower than the regulating mass, we will try the following ansatz (for the details, please refer to T.R. Morris in [11])

$$
\tilde{S}_{M}\left(V, \varphi, \bar{\varphi}, \varphi_{i}, \bar{\varphi}_{i}\right) \simeq \int d^{4} x d^{2} \theta V_{M}\left(V, \varphi, \varphi_{i}\right)+h . c .,
$$

where $V_{M}$ is assumed to be an holomorphic function of the chiral fields. The initial condition, $V_{M_{0}}$, can be read off from $S_{\mathcal{N}=4}$

$$
V_{M_{0}}\left(V, \varphi, \varphi_{i}\right)=\int i \alpha f_{a b c} \varphi^{a} \varphi_{1}^{b} \varphi_{2}^{c}=\int \alpha(-\hat{F} \cdot \vec{\varphi})_{a b} \varphi_{1}^{a} \varphi_{2}^{b},
$$

${ }^{2}$ K.Y. is grateful to T.Eguchi for pointing out this problem in [1]. 
where $\alpha=\sqrt{2} N \Re e\left(\frac{1}{g_{0}^{2}}\right)$ and $\hat{F}$ represents the set of hermitian generators of $S U(N)$ in the adjoint representation.

As was shown in [1], Polchinski's equation can be formally solved by the integral form

$$
\begin{aligned}
& \exp i \tilde{S}_{M}\left(V, \varphi, \bar{\varphi}, \varphi_{i}, \bar{\varphi}_{i}\right)=\left\{\int \prod _ { i = 1 } ^ { 2 } \mathcal { D } [ \varphi _ { i } ^ { \prime } ] \mathcal { D } [ \overline { \varphi } _ { i } ^ { \prime } ] \operatorname { e x p } i \left[\tilde{S}_{M_{0}}\left(V, \varphi, \bar{\varphi}, \varphi_{i}^{\prime}, \bar{\varphi}_{i}^{\prime}\right)+\right.\right. \\
& \left.\left.+\frac{\widetilde{M}}{2} \int \sum_{i=1}^{2}\left(\varphi_{i}^{\prime}-\varphi_{i}\right)^{2}+\text { h.c. }\right]\right\}\left\{\int \prod_{i=1}^{2} \mathcal{D}\left[\varphi_{i}^{\prime}\right] \mathcal{D}\left[\bar{\varphi}_{i}^{\prime}\right] \exp i\left[\frac{\tilde{M}}{2} \int \sum_{i=1}^{2} \varphi_{i}^{\prime 2}+\text { h.c. }\right]\right\}^{-1},
\end{aligned}
$$

where the "reduced" mass $\widetilde{M}$ is defined by $\widetilde{M}^{-1} \doteq M_{0}^{-1}-M^{-1}$. The expression (3.2) is formal in that the convergence of the r.h.s. is not well established.

In the LPA, $\tilde{S}_{M_{0}}$ is to be replaced by eq. (3.1), which is a quadratic form in the integration variables, $\varphi_{i}$,

$$
\tilde{S}_{M_{0}}\left(V, \varphi, \bar{\varphi}, \varphi_{i}^{\prime}, \bar{\varphi}_{i}^{\prime}\right)=\frac{1}{2} \int \varphi_{i a}^{\prime} \hat{A}_{i a, j b}(\varphi) \varphi_{j b}^{\prime}+h . c .
$$

with $\hat{A}_{i a, j b}(\varphi) \doteq-\alpha \epsilon_{i j}(\hat{F} \cdot \vec{\varphi})_{a b}$.

Hence the Gaussian integral

$$
\begin{aligned}
Z^{\prime}= & \left\{\int \prod_{i=1}^{2} \mathcal{D}\left[\varphi_{i}^{\prime}\right] \mathcal{D}\left[\bar{\varphi}_{i}^{\prime}\right] \exp i\left[\frac{1}{2} \int \varphi^{\prime T} \hat{A}(\varphi) \varphi^{\prime}+\frac{\widetilde{M}}{2} \int \sum_{i=1}^{2}\left(\varphi_{i a}^{\prime}-\varphi_{i a}\right)^{2}+\text { h.c. }\right]\right\} \times \\
& \times\left\{\int \prod_{i=1}^{2} \mathcal{D}\left[\varphi_{i}^{\prime}\right] \mathcal{D}\left[\bar{\varphi}_{i}^{\prime}\right] \exp i\left[\frac{\tilde{M}}{2} \int \sum_{i=1}^{2} \varphi_{i a}^{\prime 2}+\text { h.c. }\right]\right\}^{-1}
\end{aligned}
$$

is to be evaluated.

Transforming the r.h.s. of eq. (3.4) by quadrature yields

$$
\begin{aligned}
& Z^{\prime}=\exp i \frac{\widetilde{M}}{2}\left[\int \varphi_{i a}\left(1-\widetilde{M}(\hat{A}+\widetilde{M} \mathbb{1})^{-1}\right)_{i a, j b} \varphi_{j b}+\text { h.c. }\right] \times \\
& \times\left\{\int \prod_{i=1}^{2} \mathcal{D}\left[\varphi_{i}^{\prime}\right] \mathcal{D}\left[\bar{\varphi}_{i}^{\prime}\right] \exp \frac{i}{2}\left[\int \varphi_{i a}^{\prime}(\hat{A}+\widetilde{M} 1)_{i a, j b} \varphi_{j b}^{\prime}+\text { h.c. }\right]\right\} \times \\
& \times\left\{\int \prod_{i=1}^{2} \mathcal{D}\left[\varphi_{i}^{\prime}\right] \mathcal{D}\left[\bar{\varphi}_{i}^{\prime}\right] \exp \frac{i}{2}\left[\int \sum_{i=1}^{2} \widetilde{M} \varphi_{i a}^{\prime 2}+\text { h.c. }\right]\right\}^{-1} .
\end{aligned}
$$

The ratio of the Gaussian integrals in eq. (3.5) can be simply computed by applying the matrix rescaling anomaly [7]. Writing

$$
\varphi_{i a}^{\prime}=\left[\widetilde{M}(\hat{A}+\widetilde{M} \mathbb{1})^{-1}\right]_{i a, j b}^{\frac{1}{2}} \varphi_{j b}^{\prime \prime}=\left[1-\left(1-\frac{M}{M_{0}}\right) \frac{\hat{A}}{M}\right]_{i a, j b}^{-\frac{1}{2}} \varphi_{j b}^{\prime \prime},
$$

one has

$$
\prod \mathcal{D}\left[\varphi_{i}^{\prime}\right]=\prod \mathcal{D}\left[\varphi_{i}^{\prime \prime}\right] \exp i\left[\frac{1}{128 \pi^{2}} \int \operatorname{tr}\left\{\log \left(\mathbb{1}-\left(1-\frac{M}{M_{0}}\right) \frac{\hat{A}}{M}\right) W^{2}\right\}+\text { h.c. }\right]
$$


The kinematical term for the vector superfield in $S_{M}$ takes the form

$$
\frac{1}{16} \int\left[\left(\frac{1}{g_{0}^{2}}+\frac{2 N}{8 \pi^{2}} \log \frac{M}{M_{0}}\right) W_{\alpha}^{a} W^{a \alpha}+\frac{1}{8 \pi^{2}} \operatorname{tr}\left\{\log \left(1-\left(1-\frac{M}{M_{0}}\right) \frac{\hat{A}}{M}\right) W^{2}\right\}\right]+\text { h.c. }
$$

Since we are interested in the low energy dynamics only, we will restrict ourselves to massless configurations. In the $S U(2)$ case, this amounts to choosing the particular configuration $\vec{V}=(0,0, V)$ and $\vec{\varphi}=(0,0, \varphi)$. For such $S U(2)$ configuration, eq. (3.6) becomes

$$
\frac{1}{16} \int\left[\left(\frac{1}{g_{0}^{2}}+\frac{1}{2 \pi^{2}} \log \frac{M}{M_{0}}\right) W_{\alpha}^{a} W^{a \alpha}+\frac{1}{4 \pi^{2}} \log \left(1+\alpha^{2}\left(1-\frac{M}{M_{0}}\right)^{2} \frac{\varphi^{2}}{M^{2}}\right) W_{\alpha}^{a} W^{a \alpha}\right]+\text { h.c. }
$$

Hence, the effective action $S_{M}$ is approximately given by

$$
\begin{aligned}
& S_{M}\left(V, \varphi, \bar{\varphi}, \varphi_{i}, \bar{\varphi}_{i}\right)=\frac{1}{16} \int\left[\left(\frac{1}{g_{0}^{2}}+\frac{1}{2 \pi^{2}} \log \frac{M}{M_{0}}\right) W_{\alpha}^{a} W^{a \alpha}+\frac{1}{4 \pi^{2}} \log \left(1+\alpha^{2}\left(1-\frac{M}{M_{0}}\right)^{2} \times\right.\right. \\
& \left.\left.\times \frac{\varphi^{2}}{M^{2}}\right) W_{\alpha}^{a} W^{a \alpha}\right]+ \text { h.c. }+\frac{\widetilde{M}}{2}\left[\int \varphi_{i a}\left(1-\left(1-\left(1-\frac{M}{M_{0}}\right) \frac{\hat{A}}{M}\right)^{-1}\right)_{i a, j b} \varphi_{j b}+\text { h.c. }\right] .
\end{aligned}
$$

Let us now specialise to the weak coupling regime, i.e. $\frac{1}{g_{0}^{2}}>>1$. It is convenient to introduce the dynamically generated scale $\Lambda$, defined as $\Lambda=M_{0} \exp -\frac{2 \pi^{2}}{g_{0}^{2}}$, such that $\frac{1}{g^{2}(\Lambda)}=\frac{1}{g_{0}^{2}}+\frac{1}{2 \pi^{2}} \log \frac{\Lambda}{M_{0}}=0$, i.e. the running coupling diverges at $M=\Lambda$.

When $\frac{1}{g_{0}^{2}}$ is much bigger than $1, \Lambda$ is much smaller than $M_{0}$. Moreover, if one replaces the field variable $\varphi$ with its vacuum expectation value (vev), then one can also assume $|\varphi|>>\Lambda$. In this regime the quantum partition function of the mass deformed theory is given by

$$
Z_{\Lambda} \simeq \int \mathcal{D}[V] \mathcal{D}[\varphi] \mathcal{D}[\bar{\varphi}] \prod_{i=1}^{2} \mathcal{D}\left[\varphi_{i}\right] \mathcal{D}\left[\bar{\varphi}_{i}\right] \exp i\left[S_{\Lambda}+\frac{\Lambda}{2} \int \sum_{i=1}^{2} \varphi_{i}^{2}+\text { h.c. }+\cdots\right]
$$

and the form of $S_{\Lambda}$ can be read off from eq. (3.8).

The term sandwiched between the massive fields reduces to

$$
\mathbb{1}-\left(\mathbb{1}-\left(1-\frac{M}{M_{0}}\right) \frac{\hat{A}}{M}\right)^{-1} \simeq \mathbb{1}-\left(\mathbb{1}-\frac{\hat{A}}{M}\right)^{-1} \simeq-\frac{\hat{A}}{\Lambda}\left(\mathbb{1}-\frac{\hat{A}}{\Lambda}\right)^{-1} \simeq \mathbb{1},
$$

so that the last term in eq. (3.8) is given by $-\frac{\Lambda}{2} \int \sum_{i=1}^{2} \varphi_{i}^{2}+$ h.c.

Thus, for large $\left|\frac{\varphi}{\Lambda}\right|$ the total action $S_{\text {tot }}$ can be written as

$$
S_{t o t}=S_{\Lambda}+\frac{\Lambda}{2} \int \sum_{i=1}^{2} \varphi_{i}^{2}+\text { h.c. }+\cdots \simeq \frac{1}{64 \pi^{2}} \int \log \left(\frac{\varphi^{2}}{\Lambda^{2}}\right) W_{\alpha}^{a} W^{a \alpha}+\text { h.c. }+ \text { source terms. }
$$

The last expression agrees with the standard "perturbatively exact" result for $\mathcal{N}=2$ $S U(2)$ SYM. 
In [9], we have tried to interpret all the relevant part of the effective action as resulting from the effect of the Konishi anomaly or its generalisation. Compared with the present result, eqs (3.7), (3.8), the only difference is the dependence on the ratio $\frac{\varphi}{M}$. As a matter of fact, in [9] $\log \left(1+\alpha^{2}\left(1-\frac{M}{M_{0}}\right)^{2} \frac{\varphi^{2}}{M^{2}}\right)$ is replaced by $\log \left(1+\alpha^{2}\left(1-\frac{M}{M_{0}}\right)^{2} \frac{\varphi^{2}}{M_{0}^{2}}\right)$. Although it may not seem so, it makes all the difference, as the latter expression cannot be recast into the form eq. (3.9), which correctly depends on the dynamical cutoff $\Lambda$ only. Note that if one naively integrates out the massive fields in the action, eq. (2.1), neglecting the kinematical term, then one ends up with the corresponding expression $\log \left(1+\alpha^{2} \frac{\varphi^{2}}{M_{0}^{2}}\right)$.

\subsection{Generalisation to $S U(N)$}

The explicit results given for $S U(2)$ can be generalised to $S U(N)$ if the trace of the logarithmic term in eq. (3.6) is computed for the corresponding diagonal (massless) configurations. We will discuss the $S U(3)$ case first and, then, generalise the method by making use of the root equation in the Cartan-Weyl basis of the gauge group.

In the diagonal configuration, $\vec{\varphi} \cdot \hat{F}=F_{3} \varphi_{3}+F_{8} \varphi_{8}$, i.e. $\varphi_{a}$ is different from 0 for $a=3,8$ only (Cartan sub-algebra).

It is more convenient to re-express this quantity in terms of the fundamental representation, with redundant field variables,

$$
\sum_{a=1}^{8} \varphi_{a} \lambda_{a}=\operatorname{diag}\left(\hat{\varphi}_{1}, \hat{\varphi}_{2}, \hat{\varphi}_{3}\right), \quad \sum_{i} \hat{\varphi}_{i}=0
$$

with the $\lambda$ 's being the $S U(3)$ generators in the fundamental representation, normalised as $\operatorname{tr} \lambda_{a} \lambda_{b}=\frac{1}{2} \delta_{a b}$.

With this choice of gauge one has

$$
\hat{\varphi}_{1}=\frac{\varphi_{3}}{2}+\frac{\varphi_{8}}{2 \sqrt{3}}, \quad \hat{\varphi}_{2}=-\frac{\varphi_{3}}{2}+\frac{\varphi_{8}}{2 \sqrt{3}}, \quad \hat{\varphi}_{3}=-\frac{\varphi_{8}}{\sqrt{3}}
$$

and

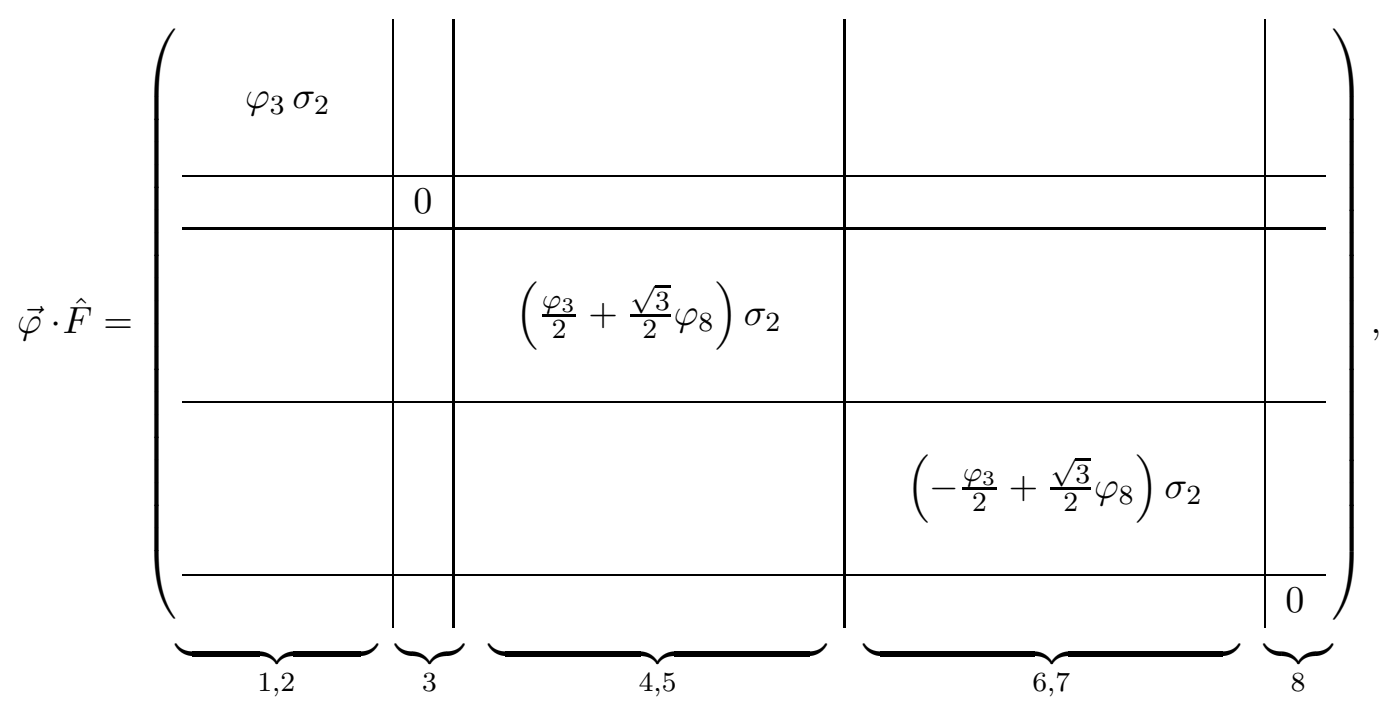


with all other elements vanishing.

All the non-trivial coefficients in the above table can be written in terms of $\hat{\varphi}_{i}-\hat{\varphi}_{j}$, namely

$$
\left(a_{1}, a_{2}, a_{3}\right) \doteq\left(\varphi_{3}, \frac{\varphi_{3}}{2}+\frac{\sqrt{3}}{2} \varphi_{8},-\frac{\varphi_{3}}{2}+\frac{\sqrt{3}}{2} \varphi_{8}\right)=\left(\hat{\varphi}_{1}-\hat{\varphi}_{2}, \hat{\varphi}_{2}-\hat{\varphi}_{3}, \hat{\varphi}_{1}-\hat{\varphi}_{3}\right) .
$$

Thus, the logarithm in eq. (3.7) can be recast as $\log \left(\mathbb{1}+\chi \sigma_{2} \otimes \varepsilon\right)$ for some constant $\chi$, and can be evaluated by using

$$
\log \left(\mathbb{1}+\chi \sigma_{2} \otimes \varepsilon\right)=\frac{1}{2 i} \log \left(\frac{1+i \chi}{1-i \chi}\right) \sigma_{2} \otimes \varepsilon+\frac{1}{2} \log \left(1+\chi^{2}\right) \mathbb{1} .
$$

Repeating the same procedure ${ }^{3}$ for $W^{2}$,

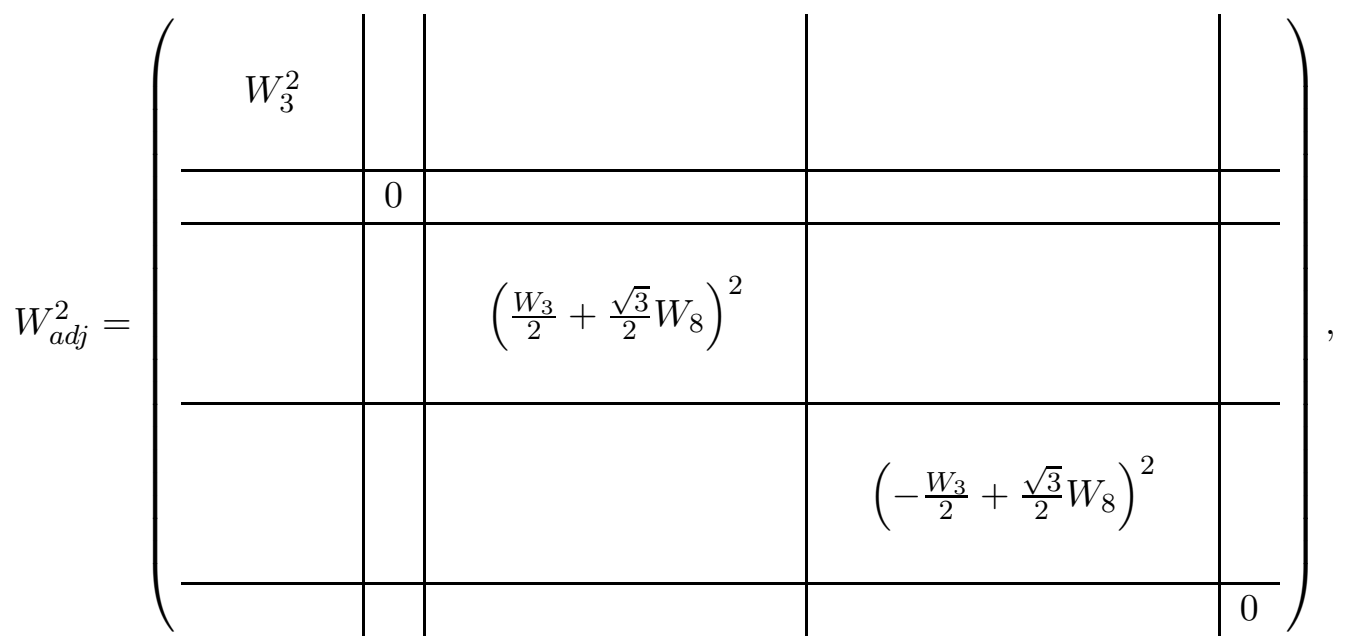

we can now take the trace over colour indices to get

$$
\begin{aligned}
& \operatorname{tr} \log \left(1+\alpha\left(1-\frac{M}{M_{0}}\right) \frac{\vec{\varphi} \cdot \hat{F}}{M} \otimes \varepsilon\right) W^{2}= \\
& =\sum_{i>j}\left(\hat{W}_{i}-\hat{W}_{j}\right)^{2} \log \left(1+\alpha^{2}\left(1-\frac{M}{M_{0}}\right)^{2}\left(\frac{\hat{\varphi}_{i}-\hat{\varphi}_{j}}{M}\right)^{2}\right) .
\end{aligned}
$$

The final expression, eq. (3.9), is therefore replaced by

$$
S_{t o t} \simeq \frac{1}{64 \pi^{2}} \int \sum_{i>j}\left(\hat{W}_{i}-\hat{W}_{j}\right)^{2} \log \left(\frac{\hat{\varphi}_{i}-\hat{\varphi}_{j}}{\Lambda}\right)^{2},
$$

$\Lambda$ being the dynamically generated cutoff.

In the $S U(N)$ case, the matrices $F_{3}, F_{8}$ and $\left\{F_{i}\right\}_{i \neq 3,8}$ generalise to the hermitian $\left\{H^{i}\right\}_{i=1}^{r}$, where $r=\operatorname{rank}(G)$ and to the complex $\left\{E^{\alpha}\right\}, \alpha=1, \ldots, \operatorname{dim}(G)-r$ respectively.

The commutators between $H^{i}$ 's and $E^{\alpha}$ 's are given by the root equation

$$
\left[H^{i}, E^{\alpha}\right]=\alpha^{i} E^{\alpha}, \quad 1 \leq i \leq r .
$$

\footnotetext{
${ }^{3}$ Again only the non-vanishing elements will be written down.
} 
In the case of $S U(N), r=N-1$ and $\operatorname{dim}(G)-r=N(N-1)$.

Let us now introduce the hermitian matrices $A^{\alpha}$ and $B^{\alpha}$, defined by

$$
E^{\alpha}=A^{\alpha}+i B^{\alpha}, \quad\left(E^{\alpha}\right)^{*}=E^{-\alpha}=A^{\alpha}-i B^{\alpha} .
$$

The root equation in terms of $A^{\alpha}$ 's and $B^{\alpha}$ 's reads

$$
\left[H^{i}, A^{\alpha}\right]=i \alpha^{i} B^{\alpha}, \quad\left[H^{i}, B^{\alpha}\right]=-i \alpha^{i} A^{\alpha},
$$

the total number of different $\left(E^{\alpha}, E^{-\alpha}\right)$ or $\left(A^{\alpha}, B^{\alpha}\right)$ pairs being $\frac{1}{2} N(N-1)$.

As in the previous section, we specialise to the diagonal configuration, $\sum_{i} H^{i} \varphi_{i}$, which in the fundamental representation can be written as a $N \times N$ diagonal matrix, $\operatorname{diag}\left(\hat{\varphi}_{1}, \ldots, \hat{\varphi}_{N}\right)$, with $\sum_{a} \hat{\varphi}_{a}=0$.

In order to evaluate that matrix element in the adjoint representation, we can read off the relevant structure constants from eq. (3.12). Writing $B^{\alpha}$ as $A^{\bar{\alpha}}$ and $\left[H^{i}, A^{\alpha(\bar{\alpha})}\right]=$ $i C^{i \alpha(\bar{\alpha}) \beta} A^{\beta}$, one has $C^{i \alpha(\bar{\alpha}) \beta}=0$ unless $\beta=\bar{\alpha}(\alpha), C^{i \alpha \bar{\alpha}}=-C^{i \bar{\alpha} \alpha}=\alpha^{i}$.

Thus the non-trivial matrix elements of the Cartan sub-algebra in the adjoint representation are given by $H_{\alpha \bar{\alpha}}^{i}=-H_{\bar{\alpha} \alpha}^{i}=-i \alpha^{i}$. This means that $\vec{\varphi} \cdot \hat{H}$ can be recast as

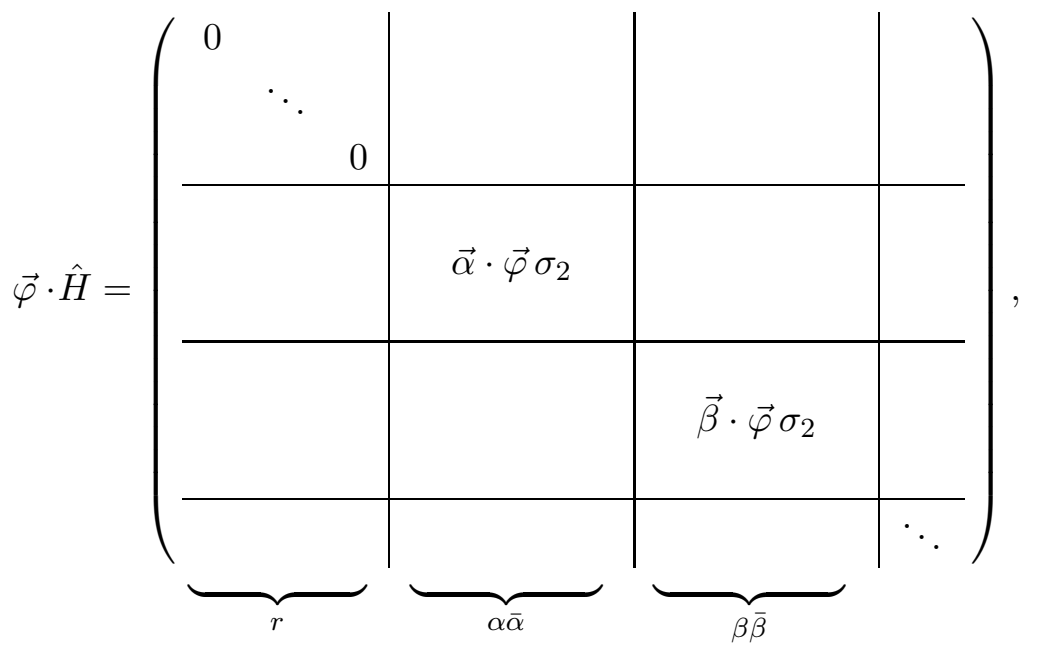

again with all other elements vanishing.

In order to find out what the values of $\vec{\alpha} \cdot \vec{\varphi}$ are, we can make use of eq. (3.12) as applied to the fundamental representation of $S U(N):(\vec{\varphi} \cdot \hat{H})_{a b}=\hat{\varphi}_{a} \delta_{a b}$ yields

$$
\left(\hat{\varphi}_{a}-\hat{\varphi}_{b}\right) E_{a b}^{\alpha}=(\vec{\alpha} \cdot \vec{\varphi}) E_{a b}^{\alpha},
$$

i.e.

i. $E_{a b}^{\alpha}=0$ unless the indices $(a, b)$ are such that $\left(\hat{\varphi}_{a}-\hat{\varphi}_{b}\right)=\vec{\alpha} \cdot \vec{\varphi}$;

ii. for a given $\vec{\alpha}$, there is at least one pair of indices $(a, b)$ such that $\left(\hat{\varphi}_{a}-\hat{\varphi}_{b}\right)=\vec{\alpha} \cdot \vec{\varphi}$;

iii. for different roots $\vec{\alpha}, \vec{\beta}$, excluding the case $\vec{\alpha}=-\vec{\beta}$, the pairs of indices in ii. are different to each other. 
As a consequence, when $\vec{\alpha}$ goes over all the $\left(E^{\alpha}, E^{-\alpha}\right)$ pairs, the corresponding $\left(\hat{\varphi}_{a}-\hat{\varphi}_{b}\right)$ takes all the different index pairs. Since the number of different $\left(E^{\alpha}, E^{-\alpha}\right)$ pairs equals that of $(a, b)$ combinations, one can rewrite eq. (3.13) as

$$
\vec{\varphi} \cdot \hat{H}=(0)^{r} \otimes \prod_{\alpha}(\vec{\alpha} \cdot \vec{\varphi}) \sigma_{2}=(0)^{r} \otimes \prod_{a>b}\left(\hat{\varphi}_{a}-\hat{\varphi}_{b}\right) \sigma_{2}
$$

These results can be used to evaluate the trace of the logarithmic term in eq. (3.7) once $\hat{F}$ has been replaced by $\hat{H}$. Making use of eq. (3.11) and writing $W^{2}=(0)^{r} \otimes$ $\prod_{a>b}\left(\hat{W}_{a}-\hat{W}_{b}\right)^{2} \mathbb{1}$ one gets

$$
\begin{aligned}
& \sum_{a>b}\left(\hat{W}_{a}-\hat{W}_{b}\right)^{2} \log \left(1+\gamma^{2}\left(1-\frac{M}{M_{0}}\right)^{2}\left(\frac{\hat{\varphi}_{a}-\hat{\varphi}_{b}}{M}\right)^{2}\right)= \\
& =\sum_{\alpha}(\vec{\alpha} \cdot \vec{W})^{2} \log \left(1+\gamma^{2}\left(1-\frac{M}{M_{0}}\right)^{2}\left(\frac{\vec{\alpha} \cdot \vec{\varphi}}{M}\right)^{2}\right)
\end{aligned}
$$

where the constant $\alpha$ in the definition of $\hat{A}(\varphi)$ [cf. eq. (3.3)] has been replaced by $\gamma$ to avoid confusion with $\vec{\alpha}$, defined by the root equation.

Hence the $W^{2}$-term in the effective action $S_{M}$ is

$$
\begin{aligned}
& \frac{1}{16} \int\left(\frac{1}{g_{0}^{2}}+\frac{N}{4 \pi^{2}} \log \left(\frac{M}{M_{0}}\right)\right) W_{\alpha}^{a} W^{a \alpha}-\frac{1}{16} \frac{1}{4 \pi^{2}} \sum_{a>b} \int\left(\hat{W}_{a}-\hat{W}_{b}\right)^{2} \times \\
& \times\left(1+\gamma^{2}\left(1-\frac{M}{M_{0}}\right)^{2}\left(\frac{\hat{\varphi}_{a}-\hat{\varphi}_{b}}{M}\right)^{2}\right) .
\end{aligned}
$$

\section{Conclusion and comments}

The results given in the previous section represent the first application of the method proposed in [1] beyond those already given in [2, 3], i.e. the exact NSVZ beta-functions.

The local potential approximation adopted here is of course expected to give only a rough estimate of the holomorphic part of the effective action, $S_{M}$. Moreover, it explicitly breaks $\mathcal{N}=2$ supersymmetry since one cannot treat the non-holomorphic kinematical terms for chiral superfields on the same footing as the kinematical term for the vector field.

Nevertheless, eq. (3.9) seems to indicate that the ERG equation applied to the mass deformed $\mathcal{N}=4 \mathrm{SYM}$ retains, at least, some correct information about the low energy behaviour of $\mathcal{N}=2 \mathrm{SYM}$. The same method can be applied to other models such as $\mathcal{N}=1^{*}$.

In order to see whether non-perturbative corrections (instanton contributions) [12, 13] too can be obtained in this way or not, one needs a more accurate estimate of the solution of Polchinski's equation.

Actually, except in the infinite $\frac{\varphi}{\Lambda}$ limit, the heavy field dependent residual potential in eq. (3.8) produces also some corrections of order $\left(\frac{\Lambda}{\varphi}\right)^{2 n}, n \in \mathbb{N}$, but they are unlikely to be instanton effects, as they should be of order $\left(\frac{\Lambda}{\varphi}\right)^{4 n}, n \in \mathbb{N}$ being the instanton number.

It is also conceivable that we should consider corrections to the Konishi anomaly itself beyond what has been computed in [7], that is the anomalous Jacobian determinant 
in presence of an external vector field, just like the chiral anomaly. This would be the "quantum anomaly" discussed in [14], but at present we do not understand the consistent way of calculating such a correction.

\section{Acknowledgments}

The authors wish to thank their colleagues for useful discussions and encouragement, in particular M. Bianchi, N. Evans, K. Konishi, S. Kovacs, T.R. Morris, G.C. Rossi and Y. Stanev.

KY wishes to thank T. Eguchi, K. Fujikawa, H. Kawai and in particular Y. Nomura for constructive comments. He also wishes to thank Prof. Fujikawa for the hospitality at the Department of Physics, University of Tokyo, where part of the work has been carried out.

\section{References}

[1] S. Arnone, C. Fusi and K. Yoshida, J. High Energy Phys. 0002 (1999) 022, hep-th/9812022.

[2] N. Arkani-Hamed and H. Murayama, Phys. Rev. D 57 (1998) 6638, hep-th/9705189.

[3] N. Arkani-Hamed and H. Murayama, J. High Energy Phys. 0006 (2000) 030, hep-th/9707133.

[4] K. G. Wilson, Phys. Rev. D 10 (1974) 2445;

K. G. Wilson in New Phenomena in Subnuclear Physics (Erice '75), ed. A. Zichichi (Plenum Press, New York, 1977);

K. G. Wilson in Recent Developments in Gauge Theories (Cargese '79), ed. G. 't Hooft (Plenum Press, New York, 1980).

[5] J. Polchinski, Nucl. Phys. B 231 (1984) 269.

G. Gallavotti, Rev. Mod. Phys. 57 (1985) 471.

[6] D. R. T. Jones, Phys. Lett. B 123 (1983) 45.

V. A. Novikov, M. A. Shifman, A. I. Vainshtein and V. I. Zakharov, Nucl. Phys. B 229 (1983) 381.

[7] K. Konishi and K. Shizuya, Nuovo Cim. A90 (1985) 111.

[8] For the problems concerning the finiteness of $\mathcal{N}=4$ supersymmetric Yang-Mills theory, see: S. Kovacs, "A perturbative re-analysis of $\mathrm{N}=4$ supersymmetric Yang-Mills theory", hep-th/9902049; PhD Thesis, April 1999, hep-th/9908171, as well as the references therein.

[9] S. Arnone, S. Chiantese and K. Yoshida, Int. J. Mod. Phys. A 16 (2001) 1811, hep-th/0012111.

[10] C. Becchi, "On the costruction of renormalized quantum field theory using renormalization group techniques," lectures given at the Parma theoretical Physics Seminar, Sept 1991. (Eds M. Bonini, G. Marchesini and E. Onofri). Revised and issued as hep-th/9607188.

[11] C. Wetterich, Phys. Lett. B 301 (1993) 90.

T.R. Morris, Int. J. Mod. Phys. A 9 (1994) 2411, hep-ph/9308265.

[12] N. Seiberg, Phys. Lett. B 206 (1988) 75. 
[13] N. Seiberg and E. Witten, Nucl. Phys. B 426 (1994) 19 [Erratum-ibid. B430 (1994) 485], hep-th/9407087; Nucl. Phys. B 431 (1994) 484, hep-th/9408099.

[14] P. I. Pronin and K. V. Stepanyantz, "Anomalies and nonperturbative results," hep-th/9803185. 